\title{
Advanced silicon processing for active planar photonic devices
}

\author{
Michael Shearn, ${ }^{a}$ Kenneth Diest, Xiankai Sun, Avi Zadok, Harry Atwater, \\ Amnon Yariv, and Axel Scherer \\ Thomas J. Watson Laboratory, California Institute of Technology, 1200 E. California Blvd., Pasadena, \\ California 91125
}

(Received 8 July 2009; accepted 5 October 2009; published 7 December 2009)

\begin{abstract}
Using high quality, anisotropically etched $\mathrm{Si}$ waveguides bonded to InGaAsP, the authors demonstrate a hybrid laser, whose optical profile overlaps both Si and III-V regions. Continuous wave laser operation was obtained up to $45^{\circ} \mathrm{C}$, with single facet power as high as $12.7 \mathrm{~mW}$ at $15^{\circ} \mathrm{C}$. Planar Si optical resonators with $Q=4.8 \times 10^{6}$ are also demonstrated. By using a $\mathrm{SF}_{6} / \mathrm{C}_{4} \mathrm{~F}_{8}$ reactive ion etch, followed by $\mathrm{H}_{2} \mathrm{SO}_{4} / \mathrm{HF}$ surface treatment and oxygen plasma oxide, the optical losses due to the waveguide and the bonding interface are minimized. Changes of optical confinement in the silicon are observed due to waveguide width variation. () 2009 American Vacuum Society.
\end{abstract}

[DOI: $10.1116 / 1.3256649]$

\section{INTRODUCTION}

Integrating silicon electronics with other materials systems is an important way to extend the capabilities of the microelectronics platform. Hybrid systems enable applications that were previously impossible or ineffective in silicon, such as lasers ${ }^{1}$ and biosensing. ${ }^{2}$ Hybrid optical systems are of particular interest, as they use silicon's capability for low loss passive waveguides ${ }^{3}$ but use other materials to avoid functionality limitations from silicon's indirect bandgap. However, direct integration through epitaxy of these systems is problematic due to differences in the lattice constant and thermal expansion coefficients. ${ }^{4}$ Recently, waferbonded III-V materials on $\mathrm{Si}$ waveguides have produced lasers ${ }^{1,5}$ and modulators. ${ }^{6}$ The bonded structure is designed to support a joint optical mode, whose profile overlaps both materials. This architecture is a promising step toward monolithic integration of electronics with optics.

In this article, we demonstrate operation of a hybrid $\mathrm{Si} / \mathrm{InGaAsP}$ Fabry-Pérot laser and describe the processing steps and optimization in its production. Si waveguide quality is maximized using a low damage, high fidelity reactive ion etch, and subsequent surface treatment, characterized by $\mathrm{Si}$ ring resonator measurements. Low temperature, plasmaassisted wafer bonding ${ }^{7}$ is then used to integrate the two material systems. Finally, optical and electrical measurements are made to characterize the hybrid laser.

\section{FABRICATION METHODS}

\section{A. Pattern definition and plasma etching}

Waveguide patterns were defined on silicon-on-insulator (SOI) wafers with a Si layer thickness from 220 to $900 \mathrm{~nm}$ on top of a $2 \mu \mathrm{m} \mathrm{SiO}_{2}$ buried oxide layer using electron beam lithography. Zeon ZEP520A was spun as $5000 \mathrm{rpm}$ and baked at $180{ }^{\circ} \mathrm{C}$ for $20 \mathrm{~min}$ before exposure with a

\footnotetext{
${ }^{a)}$ Electronic mail: mshearn@caltech.edu
}

$100 \mathrm{keV}$ electron beam. After development, patterns were placed in an oven at $150{ }^{\circ} \mathrm{C}$ for 5 min to cause the resist to reflow, reducing pattern roughness.

The waveguide patterns were transferred using an anisotropic dry etch in an inductively coupled plasma reactive ion etcher (Oxford Instruments PlasmaLab System 100 ICP-RIE 380), with a typical etch profile seen in Fig. 1. We utilized a mixed mode plasma etch using $\mathrm{SF}_{6}$ (an etch gas) and $\mathrm{C}_{4} \mathrm{~F}_{8}$ (a polymer source gas), with process conditions as described in other publications. ${ }^{8}$ In contrast to more traditional chopped processes such as the Bosch etch, ${ }^{9}$ simultaneous etching and sidewall passivation offers improved sidewall smoothness and reasonable etch rates at room temperature. ${ }^{10}$ Also important is the low forward rf power utilized by this etch, which reduces roughness from mask erosion. The etch depth varied in order to leave varying heights of Si remaining.

\section{B. Wafer bonding, mesa definition, and metallization}

After etching, the SOI wafer was cleaned by solvents and a 3:1 mixture of $\mathrm{H}_{2} \mathrm{SO}_{4}: \mathrm{H}_{2} \mathrm{O}_{2}\left(10 \mathrm{~min}\right.$ at $\left.170{ }^{\circ} \mathrm{C}\right)$, followed immediately by a HF dip to remove chemical oxide from the SOI wafer and native oxide from the III-V wafer, respectively. The reason for this cleaning step is twofold. First, it removes any remaining organics that would interfere with bonding. Second, it chemically prepares the surface for bonding by hydrogen terminating the surface, maximizing the effect of plasma activation. ${ }^{7}$ Similar processing steps have also shown improvements in optical properties of waveguides through removal of absorbing surface states. ${ }^{11}$

After cleaning, the surfaces of the wafers were then activated through exposure to a low bias oxygen plasma and bonded together under a pressure of $0.1 \mathrm{MPa}$ at $350{ }^{\circ} \mathrm{C}$ for $1 \mathrm{~h}$. Following the bonding, the InP substrate was removed by $\mathrm{HCl}$ etching and mesa and contact structures were defined lithographically and etched. A mesa structure, centered above the Si waveguide, was formed in the InGaAsP layers using photolithography and a three-phase wet etch. The etching solutions were (i) 1:1:10 mixture of $\mathrm{H}_{2} \mathrm{SO}_{4}: \mathrm{H}_{2} \mathrm{O}_{2}: \mathrm{H}_{2} \mathrm{O}$ 


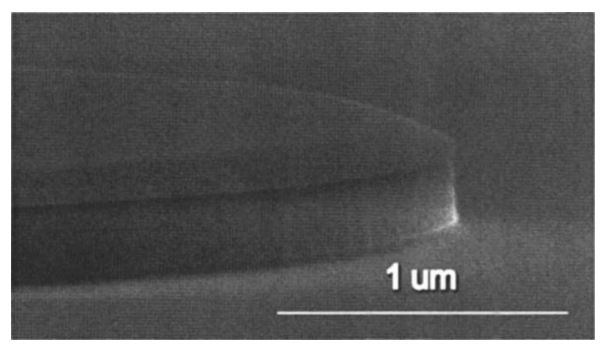

FIG. 1. Profile of a fully etched disk structure on a SOI wafer with resist. The slope of the resist is caused by reflow.

( $p$-InGaAs layer, $60 \mathrm{~s}$ ), (ii) 2:1 mixture of $\mathrm{HCl}: \mathrm{H}_{2} \mathrm{O}(p$-InP layer, $30 \mathrm{~s}$ ), and (iii) 1:1:10 mixture of $\mathrm{H}_{2} \mathrm{SO}_{4}: \mathrm{H}_{2} \mathrm{O}_{2}: \mathrm{H}_{2} \mathrm{O}$ (quaternary layers, $4 \mathrm{~min}$ ). Contacts consisting of $\mathrm{Cr} / \mathrm{AuZn} / \mathrm{Au}$ for the $p$-side metallization (on top of mesa) and $\mathrm{Cr} / \mathrm{AuGe} / \mathrm{Au}$ for the $n$-side metallization (to side of mesa) were deposited by thermal evaporation. The current flow was laterally confined by means of proton implantation at an areal dose of $5 \times 10^{14} \mathrm{~cm}^{-2}$ and an energy of $170 \mathrm{keV}$, resulting in high resistance except in a $5 \mu \mathrm{m}$ channel. Finally, the Si substrate was thinned mechanically to increase thermal conductivity to the testing chuck, resulting in a structure, as shown in Fig. 2.

\section{DEVICE MEASUREMENTS}

\section{A. Microring resonators}

To determine the compatibility of the wafer bonding chemical treatments with low loss photonic structures, we fabricated bare silicon microrings using the same processing steps used for wafer-bonded samples. The devices were fabricated from a SOI wafer with a $220 \mathrm{~nm}$ thick Si device layer on a $2 \mu \mathrm{m} \mathrm{SiO}_{2}$ buried oxide layer, and measured using evanescent coupling from a tapered fiber. ${ }^{12}$ On these devices, a thin $30 \mathrm{~nm}$ thermal oxide was grown; this differs from wafer-bonded devices which we discovered only required an ultrathin oxide formed from $\mathrm{O}_{2}$ plasma treatment.

The quality factor $Q$ of the resonator was determined experimentally from the normalized fiber transmission spectrum under weak coupling to the ring. Due to poor phase matching between the fiber and microring modes, coupling is very low even when the fiber is nearly in contact with the

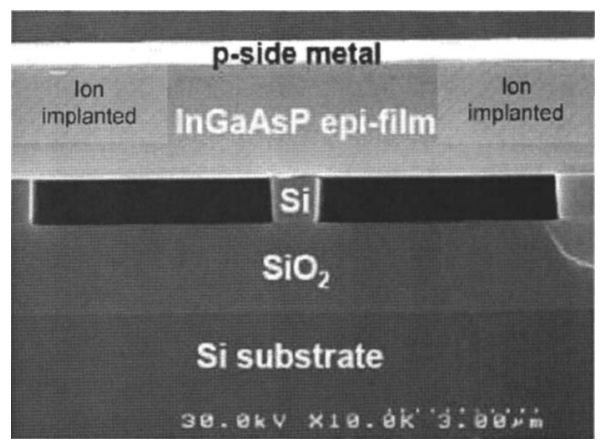

FIG. 2. Cross section of a typical bonded device. $N$-side contacts are to either side of the device (not pictured).
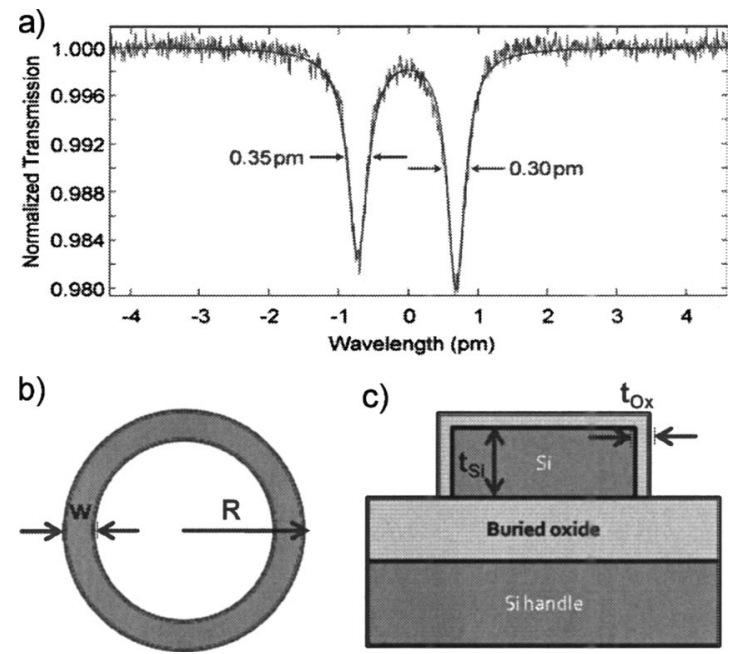

FIG. 3. Measurements and schematic of a high- $Q$ microring resonator. (a) Taper transmission versus wavelength of a doublet mode for a microring with $R=40 \mu \mathrm{m}, w=2 \mu \mathrm{m} t_{\mathrm{Si}} \approx 200 \mathrm{~nm}, t_{\mathrm{ox}} \approx 30 \mathrm{~nm}$. (b) Top view of microring. (c) Cross-sectional view of microring.

ring. However, in this regime the measured $Q$ is nearly the intrinsic $Q$. Figure 3 show results from a ring with $Q=4.1$ $\times 10^{6}$ and $Q=4.8 \times 10^{6}$ for the short and long wavelength modes, respectively. This corresponds with a loss of approximately $0.14 \mathrm{~dB} / \mathrm{cm}$. The two modes in the spectrum are a result of coupling due to scattering between the otherwise degenerate clockwise and counterclockwise circulating modes, and are only present because of the otherwise low loss of the waveguide structure. ${ }^{13,14}$

This initial result of a high quality planar structure, as opposed to freestanding geometries, ${ }^{15}$ led us to pursue waferbonded structures that incorporate gain with the waveguide.

\section{B. Hybrid lasers}

We tested Fabry-Pérot hybrid lasers with lengths from 300 to $1500 \mu \mathrm{m}$. The Si waveguides were defined on a SOI wafer with $900 \mathrm{~nm}$ thick $\mathrm{Si}$ device layer on a $2 \mu \mathrm{m} \mathrm{SiO}_{2}$ buried oxide layer. Results of a typical hybrid laser are shown in Fig. 4, which shows a device with a lasing threshold voltage $V_{\text {th }}$ of $1.3 \mathrm{~V}$ and a threshold current density $J_{\text {th }}$ of $1.25 \mathrm{kA} / \mathrm{cm}^{2}$. The maximum power output from a single fa-

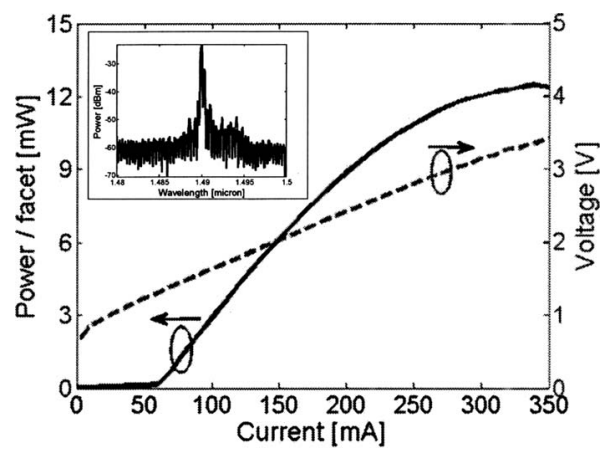

FIG. 4. $L-I-V$ curve of a $960-\mu \mathrm{m}$-long laser operating in $\mathrm{CW}$ mode at $15^{\circ} \mathrm{C}$. (Inset) Laser spectrum. 


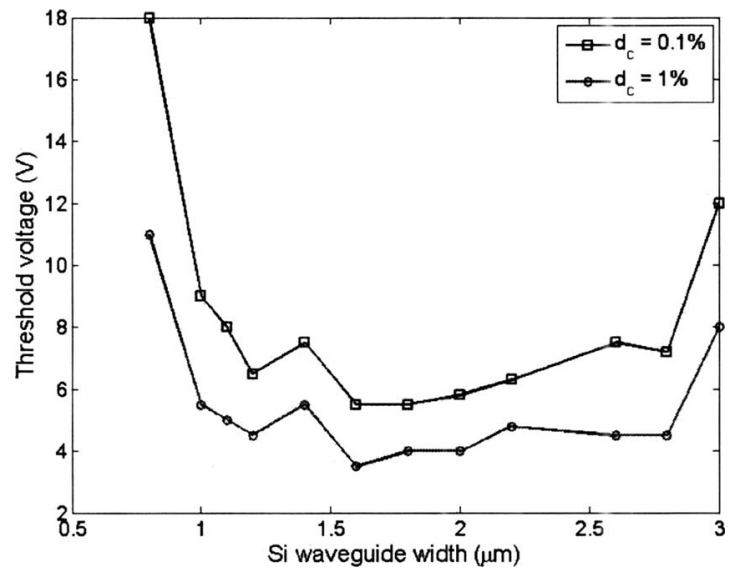

FIG. 5. Threshold voltage of hybrid lasers with various Si waveguide widths. Measurements were taken in pulsed mode at $15^{\circ} \mathrm{C}$, with $d_{c}$ denoting the duty cycle of the applied voltage.

cet was $12.7 \mathrm{~mW}$ at $15^{\circ} \mathrm{C}$. The inset of Fig. 4 shows the laser spectrum with a central wavelength of $1490 \mathrm{~nm}$.

On varying the $\mathrm{Si}$ waveguide width $w, V_{\text {th }}$ was found to have a local minimum at $w \sim 1.5 \mu \mathrm{m}$, with dependence shown in Fig. 5. This behavior can be understood qualitatively by considering the two limiting cases of waveguide width. As width decreases, less of the mode resides in the silicon, and thus experiences less feedback from the Si facets, which are of higher reflectivity than those in the III-V. On the other hand, as width increases, the mode is less confined to the quantum well region and thus requires a higher pump level to reach threshold. Further work on investigating this effect and optimizing our gain structure to utilize the relative strengths of these two regimes is underway.

\section{CONCLUSIONS}

We have shown how care in silicon processing steps for hybrid systems can lead to good optical performance. Low loss Si waveguides can be fabricated using resist reflow, lowbias dry etching, and surface chemical treatments. Subsequent integration of these optical structures at low temperature is possible with plasma-assisted wafer bonding. By using the CMOS compatible processes described, we can integrate optics with electronics seamlessly without significantly altering the constraints on upstream processing steps on the silicon. Further improvement is possible with supermode engineering ${ }^{16}$ or new materials systems, and will make this architecture even more attractive.

\section{ACKNOWLEDGMENTS}

This work was supported by Defense Advanced Research Projects Agency (DARPA) Contract No. N66001-07-1-2058 and HR0011-04-1-0054, the U.S. Air Force Office of Scientific Research (AFOSR) Grant No. FA9550-06-1-0480, and the Center for Science and Engineering of Materials, a National Science Foundation (NSF) Materials Research Science and Engineering Center at Caltech. The authors thank the Kavli Nanoscience Institute, Caltech, for supporting fabrication. M.S. thanks the NSF Graduate Research Fellowship program. A.Z. acknowledges postdoctoral fellowships from the Center for the Physics of Information, Caltech, and the Rothschild fellowship from Yad-Hanadiv Foundation, Israel.

${ }^{1}$ A. W. Fang, H. Park, O. Cohen, R. Jones, M. J. Paniccia, and J. E. Bowers, Opt. Express 14, 9203 (2006).

${ }^{2}$ R. Pantoja, J. M. Nagarah, D. M. Starace N. A. Melosh, R. Blunck, F. Bezanilla, and J. R. Heath, Biosens. Bioelectron. 20, 509 (2004).

${ }^{3}$ D. A. B. Miller, IEEE J. Sel. Top. Quantum Electron. 6, 1312 (2000).

${ }^{4}$ H. Kroemer, T.-Y. Liu, and P. M. Petroff, J. Cryst. Growth 95, 96 (1989).

${ }^{5}$ X. Sun, A. Zadok, M. J. Shearn K A. Diest, A. Ghaffari, H. A. Atwater, A. Scherer, and A. Yariv, Opt. Lett. 34, 1345 (2009).

${ }^{6}$ H.-W. Chen, Y.-h. Kuo, and J. E. , Opt. Express 16, 20571 (2008).

${ }^{7}$ D. Pasquariello and K. Hjort, IEEE J. Sel. Top. Quantum Electron. 8, 118 (2002).

${ }^{8}$ M. D. Henry, S. Walavalkar, A. Homyk, and A. Scherer, Nanotechnology 20, 255305 (2009)

${ }^{9}$ S. Rauf, W. J. Dauksher, S. B. Clemens, and K. H. Smith, J. Vac. Sci. Technol. A 20, 1177 (2002).

${ }^{10}$ H. Jansen, M. de Boer, R. Legtenberg, and M. Elwenspoek, J. Micromech. Microeng. 5, 115 (1995).

${ }^{11}$ M. Borselli, T. J. Johnson, and O. Painter, Appl. Phys. Lett. 88, 131114 (2006).

${ }^{12}$ M. Borselli, K. Srinivasan, P. E. Barclay, and O. Painter, Appl. Phys. Lett. 85, 3693 (2004).

${ }^{13}$ M. Borselli, T. Johnson, and O. Painter, Opt. Express 13, 1515 (2005).

${ }^{14}$ M. L. Gorodetsky, A. D. Pryamikov, and V. S. Ilchenko, J. Opt. Soc. Am. B 17, 1051 (2000).

${ }^{15}$ D. K. Armani, T. J. Kippenberg, S. M. Spillane, and K. J. Vahala, Nature 421, 925 (2003); M. Cai, O. Painter, and K. J. Vahala, Phys. Rev. Lett. 85, 74 (2000).

${ }^{16}$ X. Sun and A. Yariv, J. Opt. Soc. Am. B 25, 923 (2008). 